\title{
Distributed Mobile Multi-User Urban Simulation
}

\author{
Clodagh Rossi Alan Cummins Carol O’Sullivan*
}

\section{Abstract}

This sketch gives an overview of the completed and ongoing research involved in developing a 3D multi-user environment on low-end mobile devices.

\section{Introduction}

The overall aim of our research is to produce a distributed multiuser virtual model of Dublin City (Figure 1) on low-end mobile devices such as Personal Digital Assistants (PDA, Figure 2). The project uses the model developed for the Virtual Dublin Project [Hamill and O'Sullivan 2003]. There are two main areas to be considered:

- Rendering: The display of 3D models of the buildings is an area, which is very much influenced by the limited amount of memory and storage space available on the PDAs.

- Distribution: This includes the multi-user factor, which allows users that are logged onto the system to communicate with each other.

\section{Research}

To date three main areas of research have been focused upon to speed up rendering times:

- Visibility Culling: Removal of parts of a given scene if they are obscured by other buildings or are out of view.

- Level of detail Rendering: Rendering of a given model at various levels of detail allowing for a scene to be tailored for the desired frame rate.

- Impostor-based techniques: The use of flat 2D images to build up a false impression of $3 \mathrm{D}$ scene information.

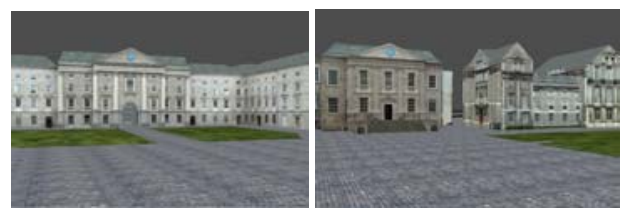

Figure 1. Screen Shots of TCD buildings

Also influencing rendering times of buildings and the correct display of mobile clients locations is the factor that networked mobile units constantly change point of access [Karimi et al. 2001]. This can result in packages requiring multiple hops via mobile clients' in the network from the point of leaving the server to being received by the required mobile client instead of the package being sent directly by the server to the client in one hop. These multiple hop packages require more time to be received and processed so that buildings are displayed in time and 'popping' effects are reduced where the buildings appear to jump on screen.

The database currently used by the server to locate and upload building data uses mobile clients' locations to determine in which section they are situated. The buildings associated with this section are then uploaded and sent to the requesting client. The tables involved include:

Section Table: Uses the client's current position to identify which section of the world it is located in.
Buildings Table: Provides the number of required buildings in a section and the associated id numbers.

Name Buildings Table: Contains the name and location of the building models.

Extra Section Table: Depending on the client's position in the world, if the client is located in but near the edge of a certain section it requires extra buildings from surrounding sections.

To reduce network traffic, a Dead Reckoning technique is used [Aronson 1997]. Dead Reckoning is a form of replicated computing in that everyone participating in a multi-user system must simulate all the entities in the environment. A predefined set of algorithms is used by all entity nodes to extrapolate the behaviour of entities in the simulation. When an agreed deviation from reality has been reached, a correction is issued.
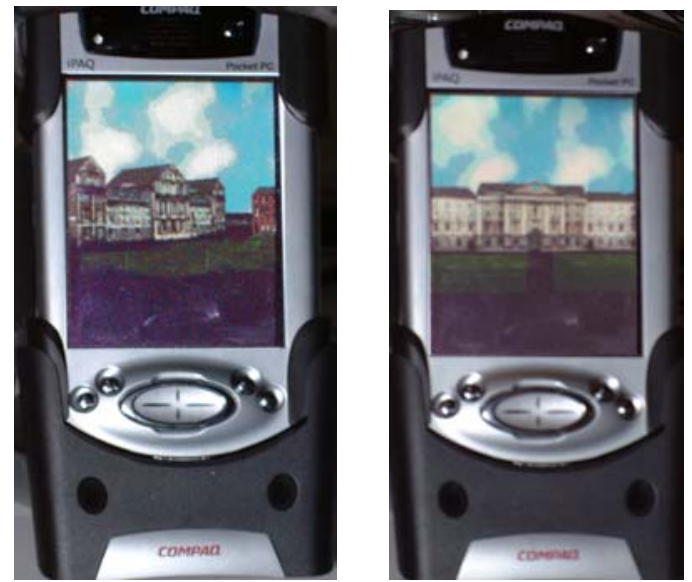

Figure 2. Screen Shots of TCD Buildings on an iPAQ

\section{Future Work}

To date an interim rendering system has been developed that allows for multiple models to be displayed on screen and multiple users to communicate. Future work in the distribution of the system will include implementation, evaluation and customisation of distributed systems algorithms and further enhancement of the Dead Reckoning algorithm. Other areas will include the development of a wireless routing algorithm that will allow the clients to remain in contact with each other and the server in an environment without a fixed network topology.

\section{References}

Hamill, J AND O'Sullivan, C. 2003. Virtual Dublin - A Framework for Real-Time Urban Simulation. In Journal of WSCG - Full Papers Vol.1-3, No. 11, ISSN 1213-6972.

Karimi, HASSAN, A. AND KRISHNAMURTHY, P. 2001. Real Time Routing in Mobile Networks using GPS and GIS techniques. At Hawaii International Conference on System Science.

ARONSON, J. 1997. Dead Reckoning: Latency Hiding for Networked Games. In Gamasutra Website:

http://www.gamasutra.com/features/19970919/aronson_01.htm.

\footnotetext{
*e-mail: rossic@cs.tcd.ie, cumminsa@cs.tcd.ie, osullica@cs.tcd.ie
} 